Desarrollo de un Sistema de Información de la Dirección de Gestión Territorial Melina Fernández

Revista ES (en y sobre Educación Superior)

Vol.1, N¹-2 / Fecha de publicación: 27/12/2021

e-ISSN: 2718-6539

https://revistas.unlp.edu.ar/ES/index

IIES - Facultad de Odontología

DOI: https://doi.org/10.24215/27186539e014

\title{
Desarrollo de un Sistema de Información de la Dirección de Gestión Territorial
}

Development of an Information System of the Directorate of Territorial Management

Desenvolvimento de um Sistema de Informação da Direcção de Gestão Territorial

\section{Licenciada Melina Fernández}

Directora de Gestión Territorial, Prosecretaría de Políticas

Sociales - UNLP

mafernandez@traajosocial.unlp.edu.ar

$\underline{0000-0001-5658-9756}$

\section{Resumen}

El Trabajo Final Integrador tiene como objetivo desarrollar un sistema de información de la Dirección de Gestión Territorial que permita respaldar la toma 
de decisiones y la gestión cotidiana de los Centros Comunitarios de Extensión Universitaria (CCEU). Enmarcado en la perspectiva de la Investigación-Gestión $(\mathrm{I}+\mathrm{G})$

Los CCEU son espacios de cogestión entre la Universidad y la comunidad donde se implementan acciones que abordan los problemas y necesidades de un territorio determinado. Dependen de la Dirección de Gestión Territorial de la Prosecretaría de Políticas Sociales de la Universidad Nacional de La Plata.

En el marco del TFI se realizó un análisis de los procedimientos e instrumentos utilizados actualmente en la DGT para la producción y gestión de información. Luego de dicho análisis, se mencionaron los nudos críticos que son el puntapié para establecer líneas de acción en el desarrollo del sistema de información.

\section{Abstract}

The objective of the Final Integrative Work is to develop an information system of the Directorate of Territorial Management that allows to support the decisionmaking and the daily management of the Community Centers of University Extension (CCEU). Framed in the perspective of Research-Management $(I+G)$ The CCEUs are spaces for co-management between the University and the community where actions are implemented that address the problems and needs of a given territory. They report to the Directorate of Territorial Management of the Assistant Secretary for Social Policies of the National University of La Plata.

Within the framework of the TFI, an analysis was carried out of the procedures and instruments currently used in the DGT for the production and management of information. After this analysis, the critical nodes that are the kick to establish lines of action in the development of the information system were mentioned. 


\section{Resumo}

O objetivo do Trabalho Integrativo Final é desenvolver um sistema de informação da Direcção de Gestão Territorial que permita apoiar a tomada de decisões e a gestão quotidiana dos Centros Comunitários de Extensão Universitária (CCEU). Enquadrado na perspectiva da Pesquisa-Gestão $(I+G)$ Os CCEUs são espaços de cogestão entre a Universidade e a comunidade onde são implementadas ações que abordam os problemas e necessidades de um determinado território. Eles se reportam à Diretoria de Gestão Territorial da Secretaria Adjunta de Políticas Sociais da Universidade Nacional de La Plata. No âmbito do TFI procedeu-se a uma análise dos procedimentos e instrumentos atualmente utilizados na DGT para a produção e gestão da informação. Após essa análise, foram mencionados os nós críticos que são o chute para estabelecer linhas de ação no desenvolvimento do sistema de informação.

\section{Palabras clave}

Gestión territorial, Prácticas universitarias, Información, sistema de información.

\section{Keywords}

Territorial management, University practices, Information, information system.

\section{Palavras chave}

Gestão territorial, práticas universitárias, informação, sistema de informação. 
El TFI presentado se realizó en base al subprograma operativo de la Universidad Nacional de La Plata Gestión de los Centros Comunitarios de Extensión Universitaria; el cual depende de la Dirección de Gestión Territorial (DGT) de la Prosecretaría de Políticas Sociales.

El funcionamiento y gestión de los CCEU se encuentra consolidado con una agenda de problemas actualizada permanentemente, con un equipo de coordinadores territoriales que realizan el seguimiento y evaluación de cada uno de los equipos universitarios que desarrollan actividades en los CCEU en el marco de la Convocatoria Específica, la convocatoria ordinaria, convocatorias de la Secretaría de Políticas Universitarias (SPU), prácticas académicas de grado, y equipos de investigación, entre otras. Asimismo, se sostienen las reuniones de inicio, término medio y de evaluación final con el objetivo de lograr un trabajo consensuado y coordinado en el territorio.

El tema que desarrolla en el TFI está relacionado con la producción y gestión de información, en relación al subprograma operativo definición de estructura de datos y mecanismos de gestión de la información; considerando que constituye una de las dificultades registradas en la DGT.

Se parte de considerar que una adecuada gestión de la información, posibilita mejorar el funcionamiento de la Dirección de Gestión Territorial y la gestión cotidiana de los CCEU; evitando de esta forma la toma de decisiones apresuradas, tardías o inconsistentes.

Spinelli plantea la disponibilidad de información como un elemento necesario para la toma de decisiones de Ixs gestores. El autor plantea que no se puede conducir a ciegas, sin datos ni información:

el gobernante y el gestor necesitan información para la toma de decisiones, la cual debe ser ágil, de rápida lectura y presentada en 
formatos amigables. Tiene que permitirle supervisar y monitorear distintos procesos para conocer lo importante y lo necesario, no lo superficial e innecesario. Por ejemplo, los anuarios estadísticos pueden alimentar la "sala de situación" del gobernante, pero no resultar prácticos para él, que necesita un tablero de control ad hoc para lo que quiere monitorear. Estos tableros tendrán pocos indicadores pero lo suficientemente sólidos como para mantener cualquier discusión y/o tomar decisiones fundadas (Spinelli, 2012, p. 119).

Entendemos que la gestión de información es una característica y una necesidad de las organizaciones en la actualidad, un recurso indispensable para el monitoreo de las operaciones cotidianas de las organizaciones y para sostener la toma de decisiones en las mismas, tal como plantea la Planificación Estratégico Situacional.

Es así que la realización del Trabajo Integrador Final pretendió abordar un tema/problema con la intención de mejorar las acciones de la Dirección de Gestión Territorial y aportar en esta línea de investigación llevada adelante por la Prosecretaría de Políticas Sociales de la UNLP.

Planteandose como objetivo desarrollar un sistema de información de la Dirección de Gestión Territorial que permita respaldar la toma de decisiones y la gestión cotidiana de los CCEU.

\section{La producción de conocimiento desde la Investigación-gestión (I+G)}

A partir de los aportes de María Bonicatto, podemos reflexionar acerca de la importancia de producir conocimiento sobre los modos de planificar y gestionar política pública. En su tesis de doctorado, la autora desarrolla el concepto de Investigación-Gestión; planteando que 
entendemos a la I+G como como el proceso mediante el cual un equipo de trabajo que tiene a cargo la gestión de política pública, se compromete a sostener un espacio de reflexión sistematizada, con un diseño metodológico que permita garantizar en simultáneo, la producción de conocimiento científico para retroalimentar y ajustar sus propios diseños de política sustantiva en el período temporal en el cual el equipo es responsable (Bonicatto, 2019, p. 89).

Se parte de considerar que todo proceso de gestión es un proceso donde se genera conocimiento y que a su vez, es importante para mejorar el proceso de gestión. Para ello se necesita además de una decisión política, sistematicidad y método. Se trata no solo de sistematizar la experiencia, sino también de reflexionar y conceptualizar sobre la misma.

Este modo específico de producción de conocimiento llamado InvestigaciónGestión o I+G incluye cinco elementos:

1) ser capaz de producir conocimiento de calidad en tiempo real, comprendido como la temporalidad marcada por los períodos de gestión

2) situacionalidad, desde la concepción matusiana de explicación situacional

3) ajuste del diseño a partir de la implementación de la política, en contraposición a la idea etapista de un diseño rígido que no se modifica

4) trazabilidad de las propuestas, concepto, utilizado para dar cuenta del proceso mediante el cual un producto es generado, nos permite sintetizar dos elementos centrales en la producción de conocimiento: la argumentación, incluyendo la validación de datos y la potencia creadora que todo proceso de producción de política pública comporta

5) alerta sobre el que no sabe que no sabe; considerando que nuestros conocimientos son por definición acotados, por lo que producir política pública 
es un ejercicio permanente de chequeo si la explicación que estamos construyendo es suficiente para poder comprender el espacio complejo sobre el cual queremos intervenir (Bonicatto, 2019, p. 433).

\section{Gestión de la información y del conocimiento}

Gestión de la información refiere a la explotación de la información para la consecución de los objetivos de la organización. Su creación, adquisición, procesamiento y difusión. La finalidad de la Gestión de la información es ofrecer mecanismos que permitan a la organización adquirir, producir y transmitir datos e informaciones con una calidad, exactitud y actualidad suficientes para servir a los objetivos de la organización.

Cuando nos referimos a Gestión del conocimiento, se considera un nivel superior, y estaría relacionado con las políticas de información, y además implica su asimilación por parte de los individuos que operan en la institución. Es así que el activo más importante de la gestión del conocimiento son las personas, que son quienes generan y acumulan ese conocimiento a partir de sus experiencias personales, y que son el elemento más importante para la institución.

Un sistema es un conjunto de elementos interrelacionados. Según Effy Oz (2008): al comprender las palabras "información" y "sistema", la definición de un sistema de información es casi intuitiva: un sistema de información está formado por todos los componentes que colaboran para procesar los datos y producir información.

El Sistema de Información (SI) puede ser definido como una colección de personas, procedimientos y equipos diseñados, construidos, operados y 
mantenidos para recoger, registrar, procesar, almacenar, recuperar y visualizar información (Teichroew, 1976 en Ros García, 1993, p. 1)

Así, se deduce que un Sistema de Información recibe datos, actúa sobre ellos y genera información; para permitir su salida al usuario, que luego podrá transformarlo en conocimiento. En una organización, un sistema de información está formado por los datos, el hardware, el software, las telecomunicaciones, las personas y los procedimientos.

En el TFI, la idea del sistema de información no se limitó a un soporte tecnológico sino que hizo referencia al proceso mediante el cual se produce y organiza la información.

\section{Presentación del proyecto y análisis situacional}

En relación al desarrollo conceptual planteado, se consideró que para poder desarrollar un sistema de información necesario realizar un análisis preliminar a partir del cual nos generamos algunos interrogantes: ¿Qué información generamos y producimos actualmente? ¿Cómo producimos la información que necesitamos? ¿Qué cuestiones registramos en los formatos actuales? ¿Con qué problemas nos encontramos? ¿Es útil esa información? ¿Cómo la utilizamos? Se intentará ir respondiendo algunas de estas preguntas a continuación.

Fernández Valdez y Ponjuán Dante (2008) nos advierten que la información y el conocimiento no solo se encuentran en los documentos o almacenes de datos, sino que también en rutinas organizativas, procesos, prácticas y normas, aunque estos no están estructurados. Es así que para gestionar información es necesario conocer con exactitud los flujos de la información dentro de la 
organización y fuera de ella, el ciclo de vida de los documentos, y el carácter de la información que se mueve en la organización, incluso de manera dispersa:

"Es evidente que la gestión de la información está relacionada con los flujos formales, es decir, a los datos e informaciones que de alguna manera están explicitados en algún soporte o sistema. Para comprender la complejidad de los flujos formales, se destacan los datos e informaciones estructurados: son sistematizados, organizados, tratados y están disponibles para su acceso; los estructurables: producidos por los propios sujetos organizacionales en los diversos sectores de la organización, pero sin selección, organización, tratamiento y acceso; y los no estructurados: son producidos por varios actores externos a la organización, y, por lo tanto, sin identificación, selección, filtración, organización, tratamiento y acceso" (Valentim, 2002, p.8 en: Valentim 2017, p1).

Como se ha mencionado anteriormente, los Centros Comunitarios basan su metodología en la Planificación Estratégica Situacional; que le otorgan complejidad al dispositivo. Se trata de un modelo de trabajo multiactoral y es en ese marco en el que se produce la información. Vemos que en el proceso existen diversas instancias de planificación colectiva, donde se construye, evalúa y ajusta el Plan Anual de Intervención de cada Centro Comunitario; mientras que el equipo de la Dirección de Gestión Territorial realiza un monitoreo territorial del proceso de forma permanente mediante Ixs coordinadores territoriales de los CCEU. 


\section{PLANIFICACIÓN Y EVALUACIÓN EL LOS CCEU}

\begin{tabular}{|c|c|c|c|}
\hline MARZo & ABRIL & JULIO & NOVIEMBRE \\
\hline $\begin{array}{c}\text { Taller de } \\
\text { Planificación } \\
\text { Estratégica }\end{array}$ & $\begin{array}{c}\text { Reunión de } \\
\text { Inicio }\end{array}$ & $\begin{array}{c}\text { Reunión de } \\
\text { corte medio }\end{array}$ & $\begin{array}{c}\text { Reunión de } \\
\text { evaluación anual }\end{array}$ \\
\hline & Monitoreo territorial del proces \\
\hline
\end{tabular}

En el marco del TFI se ha realizado un análisis detallado de la construcción de información en el marco de la propuesta metodológica de los CCEU a partir de un mapa documental con la finalidad de analizar dicho proceso ${ }^{1}$. Se trata de un tipo de mapa o gráfico que detalla los tipos de documentos que se producen o reciben en la organización; los relaciona con las funciones y actividades que los producen, e incluso con los procedimientos que los originan, e indica las unidades organizativas que intervienen en los documentos, así como los sistemas de información o aplicaciones informáticas con las que están relacionados (Bustelo, 2014).

Este mapa fue realizado sobre la base de un desarrollo anterior de la Dirección de Gestión Territorial, siendo el mismo actualizado en esta ocasión. Se tomó como base el cuadro realizado reconstruye el modelo de evaluación y seguimiento que propone el dispositivo de Centros Comunitarios.

\footnotetext{
${ }^{1}$ El mapa puede verse en la versión completa del TFI
} 
En dicho mapa, se puede ver que en las instancias de planificación estratégica que propone el dispositivo la información es co-construida mediante un formato establecido, aunque el formato del producto a lograr no está definido en un modelo de documento, mientras que en el monitoreo territorial del proceso los flujos de información son múltiples y diversos.

En un segundo momento, luego de la elaboración del mencionado mapa sobre construcción de información; se analizaron los procesos de construcción de información que están documentados, y en un tercer momento se hizo hincapié en los flujos de información que se dan en el marco del monitoreo territorial del proceso.

Se procedió a analizar cada uno de los instrumentos ${ }^{2}$ a través de diferentes variables: los actores que participan en su utilización, las dimensiones que sistematiza, el formato del documento, el carácter de la información que contiene y el análisis con respecto a su utilización.

De allí se desprenden algunas dificultades refieren a las características propias de dichos instrumentos (su formato) y otras a su utilización y apropiación por parte de los diversos actores. Reconociendo que como equipo debemos seguir trabajando sobre este último aspecto; nos centraremos ahora en las cuestiones que debemos mejorar en tanto sistema de información.

En relación a los flujos de información, nos referimos a los procesos a través de los cuales se genera la información. Los mismos se pueden representar a través de diagramas de flujos; que sirven para mostrar una secuencia de actividades, mostrar la comunicación, la transmisión de información, entre otras. De esta manera se pueden visualizar de una forma clara y precisa los

\footnotetext{
${ }^{2}$ Puede verse el análisis de cada instrumento en la versión completa del TFI
} 
procesos que conforman el sistema de información para luego determinar qué funciones hay que desarrollar.

Vamos a graficar los actores que intervienen en el proceso de construcción de información; distinguiendo entre los que pertenecen a la Dirección de Gestión Territorial y los que no; representando los procesos de comunicación y el movimiento de los datos. Este movimiento se representa por una flecha que indica la dirección de los datos.

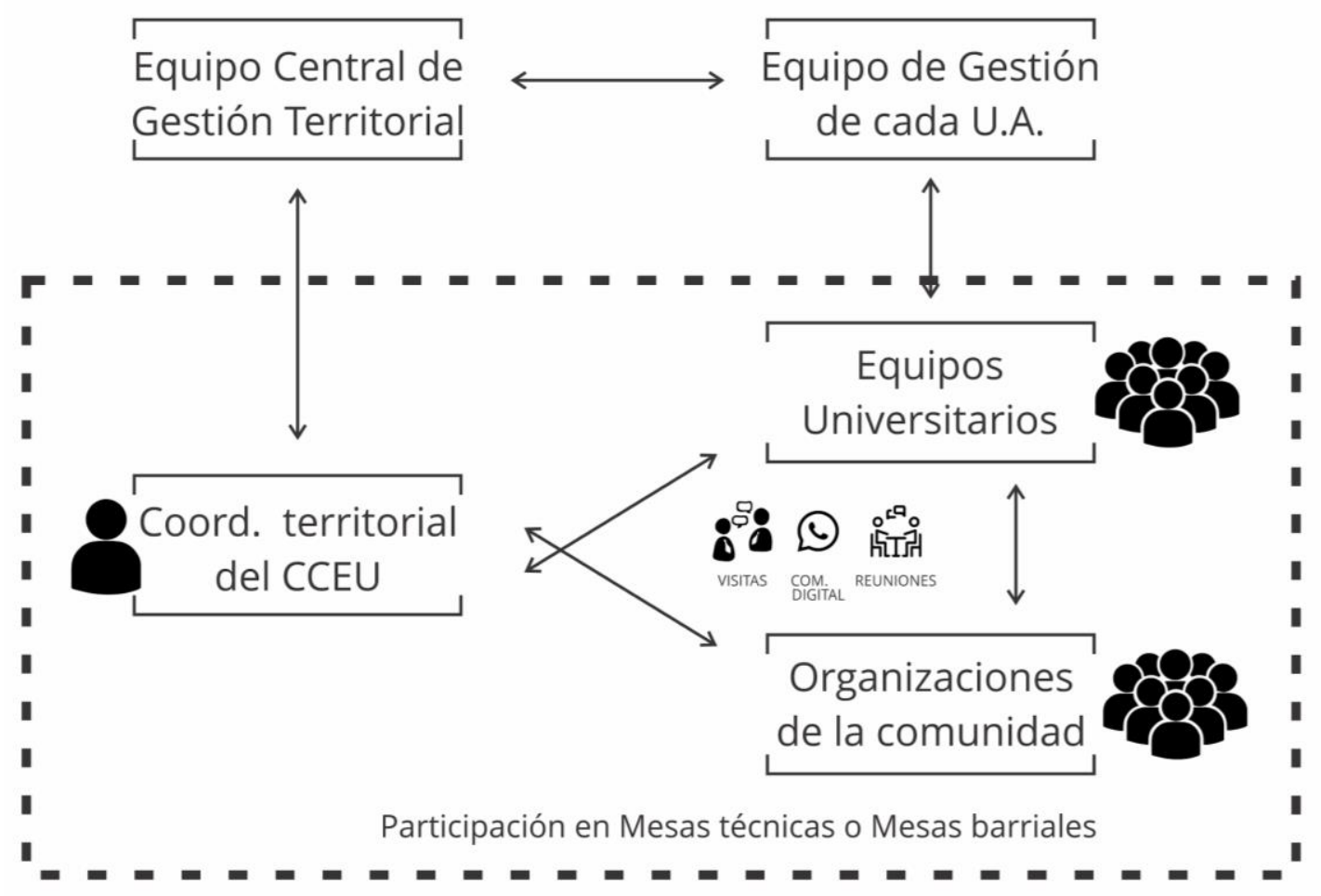

\section{Monitoreo Territorial del Proceso}

En el proceso territorial, tiene centralidad el rol de Ixs becarixs de cada CCEU, quienes tienen el rol de coordinación del Centro Comunitario. Ellxs son quienes 
conocen el barrio y tienen una mirada integral que incorpora permanentemente la percepción de las organizaciones del territorio sobre los procesos que se llevan adelante en cada barrio. $\mathrm{Y}$ ponen en marcha diversas acciones de acompañamiento y de coordinación entre diferentes actores.

El monitoreo es realizado a través de diversas estrategias como las visitas a las organizaciones y entrevistas con referentes, la presencia en actividades de los equipos universitarios, participación en mesas técnicas o barriales, reuniones, intercambios mediante comunicación digital tanto con los equipos como en los grupos de las mesas.

Analizando la complejidad de la tarea, se puede ver que resulta necesaria una adecuada estrategia de administración y gestión no solo de las tecnologías y de los procedimientos, sino de la interacción de los actores en los formatos comunicacionales que se establecen.

La experiencia nos muestra que hay información que surge en conversaciones y grupos de whatsapp o en reuniones sin ser documentada y que es parte del capital del becarix de dicho CCEU constituyendo conocimiento tácito. Asimismo, se evidencian otras dificultades relacionadas con el uso de las tecnologías de la vida cotidiana; aún no hemos elaborado directrices claras sobre cómo gestionar la información que allí se genera, por lo que cada becarix organiza sus registros de manera distinta.

\section{Algunos resultados: nudos críticos en la gestión de información}

A partir del análisis realizado, se pudieron identificar algunos nudos críticos que permitirán un acercamiento a algunas orientaciones para el desarrollo del sistema de información. 
1. Existe actualmente un cúmulo de informaciones diversas que se caracterizan por su dispersión.

En el monitoreo territorial se genera información que no se encuentra efectivamente documentada en archivos institucionales que permitan su efectivo registro y procesamiento; se encuentra almacenada en mails o teléfonos celulares de Ixs coordinadores territoriales, grupos de whatsapp, entre otras. Es necesaria una estrategia de gestión de la información que surge en la comunicación digital. Por otro lado, los instrumentos que se utilizan para la sistematización no se encuentran centralizados o en algunos casos solo se dispone de ellos en formato papel.

1. La información no está suficientemente estructurada y organizada para su acceso rápido y eficiente.

Como hemos visto, la información no es accesible: debido a la naturaleza de la misma (dispersa y no estructurada) y a su formato (en muchas ocasiones en papel dentro de expedientes) no se tiene la capacidad de estar disponible a toda hora y en cualquier lugar. En ocasiones el acceso a los datos está limitado al horario de trabajo de las personas responsables.

2. Con respecto a su uso, la totalidad de la misma es de acceso restringido.

Conviene aquí distinguir entre Información pública y privada: la información pública es aquella que la organización produce u organiza de cara al "público" exterior para comunicar hechos, actividades, acontecimientos, o datos; es decir se encuentra disponible para otros usuarios en distintos formatos y niveles de acceso. Mientras que la información privada es de carácter restringido hacia el interior de la organización. 
Actualmente la Dirección de Gestión Territorial no cuenta con mecanismos de producción de información pública o de difusión abierta de la misma. Incluso hay actores institucionales que participan en el proceso de implementación que no acceden a la información de los proyectos como es el caso de las Secretarías de Extensión.

Un dato importante respecto al segundo y tercer punto; es que si bien la Universidad cuenta con un sistema de gestión de proyectos de extensión, la Convocatoria Específica dejó de utilizarlo por no encontrarse adecuado a las necesidades de información de la Dirección. Particularmente, el sistema de evaluación es diferente a la Convocatoria Ordinaria, y los procesos de seguimiento y evaluación tienen una mayor periodicidad.

Podría considerarse que dicho sistema puede constituir la base sobre el cual se desarrolle el Sistema de Información de la Dirección de Gestión Territorial, incorporando la complejidad anteriormente descripta.

\section{Algunas líneas para el desarrollo del sistema de información}

En relación a lo anteriormente descripto en el análisis situacional, podemos concluir que el sistema de información tendría los siguientes objetivos:

- Mejorar de los canales de producción de información interna y externa

- Mejorar los canales de acceso a la información por parte de los diferentes usuarixs

- Producir información oportuna y de calidad que retroalimente los procesos de toma de decisiones de la DGT

Asimismo, en relación a los nudos críticos mencionados, el sistema debería: 
1. Permitir el ingreso de información, su almacenamiento, la consulta de la misma por los diversos actores involucrados y la extracción en forma de estadísticas.

2. Centralizar la información en un mismo sitio; siendo accesible para distintos usuarixs con distintos niveles de acceso según cada actor.

3. Establecer un canal de información pública a modo de repositorio para el acceso a todas las personas que deseen interiorizarse acerca del trabajo que se realiza en los Centros Comunitarios.

4. Visualizar en tiempo real los procesos de implementación de acciones y control de ejecución presupuestaria.

5. Identificar brechas entre lo planificado y lo efectivamente implementado

6. Otorgarle trazabilidad a la agenda de problemas, es decir permitir revisar el proceso de construcción del PAl.

\section{A modo de conclusión}

En el marco de la cursada de la Especialización en Gestión de la Educación Superior, se propuso la elaboración de un Proyecto de Intervención que se inscriba en el ámbito de desempeño en la Universidad, tendiente a la transformación o al mejoramiento de alguna dimensión de esas prácticas o del área involucrada.

Este TFI constituyó una oportunidad para abordar un tema importante como lo es la gestión de información en un dispositivo complejo que son los Centros Comunitarios de Extensión Universitaria.

Se ha podido avanzar el desarrollo conceptual de las dimensiones centrales que se abordan en el proyecto, con las dificultades que ello implicó, pues se trataba de un área temática novedosa para la autora. Asimismo, se avanzó en 
la realización un análisis situacional del estado actual del área y las prácticas de producción de información que allí se generan; estableciendo algunos nudos críticos. Por último, se describieron algunas líneas orientativas de lo que sería el Sistema.

Sin dudas, quedan muchos aspectos en los que profundizar, pero ha sido una contribución para mejorar la gestión de la información en la Dirección y con ello mejorar la gestión cotidiana de los Centros Comunitarios de la Universidad Nacional de La Plata.

\section{Bibliografía}

- Bonicatto, María (2019) Doble Vía. El Aporte de Planificación Estratégica Situacional A La Extensión Estatutaria de La UNLP. Tesis de Doctorado En Trabajo Social -en Proceso de Evaluación-.

- Bonicatto, María (2019) Gobernantes Impreparados Para Gobernar. ¿La Gestión Y La Planificación Estratégicas Son Posibles?

- Bonicatto María (2014) Recursos Críticos Para La Gestión. Administración En Trabajo Social. Fts. UNLP Material Inédito Publicado En Www.Trabajosocial.UNLP.Edu.Ar

- Bonicatto, María (2017). Gestión Estratégica Planificada (Gep). Un Método Para La Gestión En Organizaciones Públicas. Serie Libros de Cátedra. La Plata: Editorial de La UNLP (Edulp).

- Bustelo Ruesta Carlota, García Morales Huidobro E. Tendencias En La Gestión de Información, La Documentación Y El Conocimiento En Las Organizaciones. El Profesional de La Información 2001;10(12). Https:/Www.Researchgate.Net/Publication/28157393 tendencias en la 
gestion de la informacion la documentacion y el conocimiento en las organizaciones

- Fernández Valdés, María de Las Mercedes Y Ponjuán Dante, Gloria (2008). Análisis Conceptual de Las Principales Interacciones Entre La Gestión de Información, La Gestión Documental Y La Gestión del Conocimiento. Acimed.

- García, E. R. (2015). Del conocimiento tácito al conocimiento explícito: retos para la gestión del conocimiento organizacional. Informatio, 20(1), 37-48.

Recuperado de Http://Informatio.Eubca.Edu.Uy/Ojs/Index.Php/Infor/Article/Viewfile/172/2 $\underline{52}$

- Ozz, Effy. Administración de los sistemas de información, 5a. ed. Traducción de Miguel Ángel Martínez Sarmiento. Diponible en: http://cotana.informatica.edu.bo/downloads/Administracion\%20SI.pdf

- Prada Madrid, Enio (2008). Los Insumos Invisibles de decisión: Datos, Información Y Conocimiento. Anales de Documentación, 11, 183-196. Disponible En: Http://Revistas.Um.Es/Analesdoc/Article/View/24881/24191

- Valentim, M. L. P. (2017). Gestión de la información: dilemas y perspectivas. Palabra Clave (La Plata),7(1), e026. https://doi.org/10.24215/18539912e026

\section{Otras referencias:}

- Estatuto de la Universidad Nacional de La Plata. (2008). La Plata, Buenos Aires, Argentina.

- Plan Estratégico de la Universidad Nacional de La Plata 2018-2022. Disponible en www.unlp.edu.ar 
- Tauber, Fernando (2018) Pensar la Universidad. Proyecto institucional de la Universidad de La Plata 2018-2022. Publicación Institucional de la Universidad Nacional de La Plata. 1ra edición. Disponible en: http://sedici.unlp.edu.ar/handle/10915/68411

- Programa Territorial de Extensión Universitaria. 2012. Disponible en: https://unlp.edu.ar/frontend/media/53/19953/861ec2c5545d760b5b4d912 382db7be0.pdf

- Resolución UNLP 5422/19. Organigrama y responsabilidades primarias / acciones de los distintos organismos de la Presidencia de la Universidad Nacional de La Plata. 2019. 